\title{
Mitochondrion-mediated iron accumulation promotes carcinogenesis and Warburg effect through reactive oxygen species in osteosarcoma
}

Shuo $\mathrm{Ni}^{1 \dagger}$, Yanbin Kuang ${ }^{2 \dagger}$, Yin Yuan ${ }^{3 \dagger}$ and Baoqing $\mathrm{Yu}^{1 *}$ (1)

\begin{abstract}
Background: Iron metabolism disorder is closely associated with several malignant tumors, however the mechanisms underlying iron and the carcinogenesis in osteosarcoma are not yet well understood.

Methods: Cell proliferation ability of osteosarcoma cell lines was measured by CCK-8, EdU incorporation and colony formation assays. Cell cycle analysis was detected by flow cytometry. The carcinogenesis of osteosarcoma was measured by soft-agar formation, trans-well and Wound healing-scratch assay. Warburg effect was detected by Seahorse respirometry assays. Reactive oxygen species (ROS) level was measured by Dichlorodihydrofluorescein diacetate (DCFH-DA) fluorescent probes. Western blotting was used to measure the expression of mitoferrin 1 (SLC25A37) and mitoferrin 2 (SLC25A28). Iron level in vitro and vivo was detected by iron assay kit. RNAi stable cell lines was generated using shRNA.
\end{abstract}

Results: Iron promoted proliferation, carcinogenesis and Warburg effect of osteosarcoma cells. Iron-induced reactive oxygen species (ROS) played an important role in these processes. Iron accumulated more in mitochondrion than in cytoplasm, suggesting mitochondrion-mediated iron accumulation was involved in the development of osteosarcoma. Moreover, iron upregulated the expression of mitoferrin 1 (SLC25A37) and mitoferrin 2 (SLC25A28). Knockdown of mitoferrin 1 (SLC25A37) and mitoferrin 2 (SLC25A28) decreased the production of ROS. In addition, iron increased the expression of Warburg key enzymes HK2 and Glut1, and affected AMPK/mTORC1 signaling axis.

Conclusions: Mitochondrion-mediated iron accumulation promotes carcinogenesis and Warburg effect of osteosarcoma cells. Meanwhile, iron deprivation might be a novel effective strategy in the treatment of osteosarcoma.

Keywords: Iron, Mitoferrin, Warburg, Reactive oxygen species, Osteosarcoma

*Correspondence: doctorbqy@163.com

† Shuo Ni, Yanbin Kuang and Yin Yuan contributed equally to this work

1 Department of Orthopedics, Shanghai Pudong Hospital, Fudan

University Pudong Medical Center, 2800 Gongwei Road, Pudong, Shanghai 201399, China

Full list of author information is available at the end of the article

\section{Background}

Iron metabolism is of central importance to numerous biological processes, including DNA replication, cellcycle progression, electron transport chain and microsomal electron transport proteins, as well as the production of iron-sulfur proteins $[1,2]$. Iron is absorbed from the duodenum by divalent metal transporter 1 (DMT1) and then transported by transferrin, which is regulated by hepcidin and cellular iron exporter ferroportin (FPN1)

(c) The Author(s) 2020. This article is licensed under a Creative Commons Attribution 4.0 International License, which permits use, sharing, adaptation, distribution and reproduction in any medium or format, as long as you give appropriate credit to the original author(s) and the source, provide a link to the Creative Commons licence, and indicate if changes were made. The images or other third party material in this article are included in the article's Creative Commons licence, unless indicated otherwise in a credit line to the material. If material is not included in the article's Creative Commons licence and your intended use is not permitted by statutory regulation or exceeds the permitted use, you will need to obtain permission directly from the copyright holder. To view a copy of this licence, visit http://creativeco mmons.org/licenses/by/4.0/. The Creative Commons Public Domain Dedication waiver (http://creativecommons.org/publicdomain/ zero/1.0/) applies to the data made available in this article, unless otherwise stated in a credit line to the data. 
[3, 4]. Notably, mitochondrion is the main consumer of iron in cells for its function of synthesis or assemble many important proteins such as heme and iron sulfur clusters [5, 6]. Mitoferrin 1 (SLC25A37) and mitoferrin 2 (SLC25A28) are the main iron importers in mitochondrion, which controlled the iron homeostasis by transferring the ferrous iron to the mitochondrial matrix [7, 8]. Accumulating evidence indicates that mitochondrial iron accumulation is associated with human disease such as pulmonary and cardiovascular disorders [9-11]. However, little is known about the role of mitochondrion-mediated iron accumulation in the initiation and development of malignant tumors.

Several large epidemiological studies have demonstrated that iron contributes to the occurrence and progression of esophageal cancer, colorectal cancer, liver cancer, and lung cancer [12-15]. Dietary intake of highiron is associated with increased colorectal cancer risks [16]. Moreover, iron overload promotes carcinogenesis and tumorigenesis in colorectal and lung cancer [17-19]. Some studies have demonstrated that iron could induce reactive oxygen species (ROS) and promote carcinogenesis [20]. Moreover, some studies also demonstrate that mitochondrion-mediated iron-dependent ROS accumulation promotes pancreatic tumorigenesis in mice [21]. Some iron chelator such as deferoxamine (DFO), Di-2-pyridylketone-4,4-dimethyl-3-thiosemicarbazone (Dp44mT) and ciclopirox olamine (CPX) have been showed with anti-cancer effects recently [18, 22, 23].

As a hallmark of cancer cells, Warburg effect is associated with high level of glycolysis even when cancer cells are under aerobic conditions [24]. Several studies have demonstrated that reactive oxygen species (ROS) regulate Warburg effect [21, 24-26]. In the present study, we investigated the association among iron, ROS and Warburg effects in carcinogenesis of osteosarcoma. Meanwhile, our work reveals that iron deprivation might be a novel effective strategy in the treatment of osteosarcoma.

\section{Methods and materials Cell lines and reagents}

Human osteosarcoma cell line SAOS-2 and U2OS were obtained from American Type Culture Collection (ATCC, USA). Both cell lines were cultured in RPMI 1640 medium (Gibco, Gaithersburg, MD, USA) with 10\% fetal bovine serum (FBS, Invitrogen, Carlsbad, CA, USA) in a concentration of $5 \% \mathrm{CO}_{2}$ and $37^{\circ} \mathrm{C}$ incubator. Ferric ammonium citrate (FAC) and Deferoxamine (DFO) were purchased from Sigma (St. Louis, MO, USA).

\section{Mouse xenograft tumor model}

The animal experiments in this study was approved by the Animal Ethics Committee of Zhejiang University
School of Medicine and in accordance with the National Institutes of Health (NIH) Guide for the animal treatments of Laboratory Animals. Twelve BALB/c nude mice were randomly divided into 2 groups. A number of 2 * $10^{6}$ SAOS- 2 cells resuspended in $200 \mu \mathrm{FBS}$-free RPMI 1640 medium was injected subcutaneously into the arms of nude mice (6-week-old, female). Tumor volumes were measured every 2 days. Deferoxamine (DFO) $16 \mathrm{mg} /$ $\mathrm{kg}$ or normal saline (NS) was injected intraperitoneally for 2 weeks. Deferoxamine (DFO) was purchased from Sigma (St. Louis, MO, USA). After 2 weeks, all animals were sacrificed.

\section{Cell viability assay and cell cycle analysis}

Cell counting Kit-8 (CCK-8; Beyotime, Shanghai, China) was obtained from Beyotime and be used to detect the viability of two cell line according to the manufactories' protocol. FAC $(100 \mu \mathrm{M})$, DFO $((100 \mu \mathrm{M}))$ and PBS were added to different groups respectively. Then the 96-well plate was assessed at $450 \mathrm{~nm} 24 \mathrm{~h}, 48 \mathrm{~h}$ and $72 \mathrm{~h}$ after incubation. For the cell cycle assay, a total of $1 \times 10^{6}$ cells nearly $80 \%$ confluent from 6 -well plate were collected and fixed in $70 \%$ ethanol for $24 \mathrm{~h}$ at $4{ }^{\circ} \mathrm{C}$ like previously reported [27]. Cells were then washed in Phosphate Buffer Saline (PBS) and stained with propidium iodide (PI) before being analyzed by flow cytometry (BD Biosciences). G1, S and G2/M phases were calculated by Modfit Software (Verity Software House, Inc.).

\section{Plate colony formation}

Cells were collected and resuspended in RPMI 1640 medium containing $100 \mu \mathrm{M}$ FAC and $100 \mu \mathrm{M}$ DFO, respectively. Then, the cells were transferred to 6-well plates at the density of 500 cells per well and incubated for 14 days. The culture medium in different groups were replaced every 2 days until the end of this experiment. Finally, colonies were stained by $1 \%$ Giemsa stain solution (Solarbio, Beijing, China) for $30 \mathrm{~min}$. All of the colonies were counted and quantified.

\section{EdU cell proliferation assay}

Edu Cell Proliferation Kit (Beyotime, Shanghai, China) was used to detect the proliferation of two cell lines visually according to the manufactories' protocol. After being stimulated by $100 \mu \mathrm{M}$ FAC or DFO for $24 \mathrm{~h}$ respectively, cells were stained and captured by Olympus FSX100 microscope (Olympus, Tokyo, Japan).

\section{Soft-agar colony formation assay}

Two percent agar solution was made in room temperature and sterilized by pressure cooker in $120{ }^{\circ} \mathrm{C}$ for $6 \mathrm{~h}$, then it was stored in $4{ }^{\circ} \mathrm{C}$ in the refrigerator. Microwave oven was used to heat the solid agar until it was become 
liquid at $37{ }^{\circ} \mathrm{C}$. Two percent of agar solution mixed with culture medium containing $100 \mu \mathrm{M}$ FAC or $100 \mu \mathrm{M}$ DFO was made respectively. Then the mixed medium was added into 24-well plate, and moved to $4{ }^{\circ} \mathrm{C}$ refrigerator immediately until it turns into solid. Then cell suspension containing each type of cell was added on to the top of solidified agar $(500 / 50 \mu \mathrm{L})$. Thereafter, $2 \%$ agar solution containing each conditional medium $(1: 6 \mathrm{v} / \mathrm{v})$ along with Matrigel $(1: 30 \mathrm{v} / \mathrm{v})$. After 2 weeks of culture, colony numbers were counted.

\section{Trans-well assay}

Trans-well chambers (Corning Costar, Cambridge, MA, USA) were used to detect the migration and invasion ability of cells. In brief, cells were cultured in FBS-free medium overnight. Then it was transferred to the upper chambers $\left(10^{5}\right.$ cells/well $)$. Culture medium containing $100 \mu \mathrm{M}$ was added into the lower chamber, followed by a 24-h incubation. The next day, cells on the upper surface (non-migrated cells) were gently removed by small sticks. The cells on the opposite surface (migrated cells) were counted and imaged. For the invasion assay, diluted (1:6) Matrigel (BD Bioscience, San Diego, CA, USA) was used to cover the surface of upper chamber before cells being planted on it. Other steps were the same as migration assay.

\section{Wound healing-scratch assay}

SAOS-2 and U2OS cells were seeded in 12-well plates. After cells were confluence, wound area was made in cell monolayer by pipette tips. FBS-free RPMI 1640 medium containing $100 \mu \mathrm{M}$ FAC or $100 \mu \mathrm{M}$ DFO were added and incubated for $24 \mathrm{~h}$. The wound closure was captured and the percentage of arear was evaluated by ImageJ software (USA).

\section{Seahorse XF24 respirometry assay}

Seahorse Bioscience Extracellular Flux Analyzer (XF24, Seahorse Bioscience Inc., North Billerica, MA, USA) was used to detect the oxygen consuming rate (OCR), and extracellular acid rate (ECAR). Mito Stress Test Kit from Agilent was used according to the manufacturer's protocol. Briefly, $1 * 10^{4}$ cells were seeded in the 24-well plate in conditional culture medium and incubated overnight. Then the cells were washed with XF media (1\% FBS) then cultured in a $\mathrm{CO}_{2}$-free incubator at $37{ }^{\circ} \mathrm{C}$ for $2 \mathrm{~h}$. ECAR and OCR measurements were performed. OCR and ECAR were measured in a typical 8 min cycle of mix ( 2 to $4 \mathrm{~min}$ ), dwell (2 min), and measure (2 to $4 \mathrm{~min}$ ).

\section{Mitochondrial extraction}

Mitochondrial isolation and extraction were performed according to the manufacturers' protocol. Mitochondria/
Cytosol Fractionation Kits (ab65320, Abcam). Briefly, cells were harvested after culturing in different conditional medium for $24 \mathrm{~h}$. A number of $5^{*} 10^{7}$ cells was centrifuged at $600 \times g$ for $5 \mathrm{~min}$ at $4{ }^{\circ} \mathrm{C}$. Cells were resuspended with cytosol extraction buffer mix after washing with cold PBS. Then the cells were incubated $10 \mathrm{~min}$ and performed the task with grinder on ice.

\section{Iron assay}

Iron assay was performed according to the manufacturers' protocol of Iron Assay Kit (ab83366, Abcam) as previously described [21]. In brief, samples were incubated for $30 \mathrm{~min}$ at $25^{\circ} \mathrm{C}$, followed by an incubation of $60 \mathrm{~min}$ with iron probe at $25{ }^{\circ} \mathrm{C}$. Then all the samples were moved to microplate reader.

\section{Generation of RNAi stable cell lines}

Human SLC25A37 shRNA and human SLC25A28 shRNA sequences were designed by Biomics Biotech (Shanghai, China). Non-specific shRNA (NS) was used as control. Briefly, HEK293T cells were transfected by lentivirus-shRNA. After $48 \mathrm{~h}$ of incubation, culture medium containing lentivirus was used to infect SAOS-2 and U2OS cells lines. Lipofectamine 3000 was used in the transfection procedure. The transfection procedure was performed according to the manufacturers' protocol of Lipofectamine 3000 (Invitrogen, Carlsbad, CA, USA). Puromycin $(2 \mu \mathrm{g} / \mathrm{ml})$ was used to screen stable cell lines. Knockdown of SLC25A37 and SLC25A28 were confirmed by qPCR and Western blot. The most effective sequence of SLC25A37 shRNA and human SLC25A28 shRNA are listed in Additional file 1: Table S2.

\section{ROS production detection}

Dichlorodihydrofluorescein diacetate (DCFH-DA; Beyotime, Shanghai, People's Republic of China) was used to detect ROS production according to the manufacturers' protocol. Briefly, $5 * 10^{5}$ cells were planted in the 6-well plate in different conditional culture medium $(100 \mu \mathrm{M}$ FAC or $100 \mu \mathrm{M}$ DFO) for $24 \mathrm{~h}$. at the day of measurement, then the culture medium was removed. Next, FBSfree medium with DCFH-DA was added to the dish and then incubated for $20 \mathrm{~min}$. The fluorescence intensity of cells was detected by microplate reader.

\section{TCGA database and analysis}

The correlation of mitochondrion-related genes and Warburg genes was analyzed by GEPIA web tools (http:// gepia.cancer-pku.cn/) based on the TCGA database.

\section{Western blot analysis}

Cells were collected after stimulated with $100 \mu \mathrm{M}$ FAC or $100 \mu \mathrm{M}$ DFO for $24 \mathrm{~h}$. Cellular proteins were extracted 
by RIPA lysis buffer containing protease and phosphatase inhibitors. SDS-PAGE was used to separate the proteins. After running process, gels were transferred to PVDF membranes and immersed in primary antibodies. The next day, membranes were incubated with secondary antibodies and be visualized by chemiluminescence detection kit (Beyotime). Slc25a28 antibody (ab90170, 1:100) was from Abcam. antibodies specific for SLC25A37/Mitoferrin1 (26469-1-AP, 1:100) and Glut1 (66290-1-Ig, 1:100) were purchased from Proteintech. Anti-phospho-AMPK (Thr172) antibody (\#2535S, 1:100), Anti-AMPKo Antibody (\#2532, 1:100), anti-p70-S6K (9202S, 1:100), anti-phospo-p70-S6K (Thr389) (9234S, 1:100), anti-Hexokinase 2 (2867S, 1:100), anti-phospho4EBP1 (Thr70) (13396, 1:100) and anti-4EBP1 (9644s, 1:100) were from Cell Signaling Technology. Anti-PCNA (2586S, 1:100) was from Cell Signaling Technology. The anti-GAPDH antibody (BM1623, 1:1000), anti- $\beta$ actin antibody (BM0627, 1:1000), anti- $\alpha$-tubulin antibody (BM1452, 1:1000), anti-rabbit IgG-HRP antibody (BA1054, 1:5000), and anti-mouse IgG-HRP antibody (BA1050, 1:5000) were purchased from Boster Biological Technology (Wuhan, China).

\section{RNA extraction and qRT-PCR}

Cells treated with $100 \mu \mathrm{M}$ FAC or $100 \mu \mathrm{M}$ DFO for $24 \mathrm{~h}$ were collected for RNA extraction. RNeasy Mini Kit (Qiagen, Valencia, USA) was used to extract the RNA. Then it was reverse-transcribed into cDNA. The mRNA expression levels were assessed by qRT-PCR system (Applied Biosystems, Foster, CA, USA). The primers we used are listed in Additional file 1: Table S1.

\section{Statistical analysis}

All experimental data was presented as the mean \pm SD ( $\mathrm{n} \geq 3$ ). GraphPad Prism (version 7, GraphPad Software, La Jolla, CA, USA) was used to analyse the data. Student's $t$-test was used between treated and control group. One-way ANOVA was used for multiple groups. LSD-t test was applied when data needed to be compared with control in multiple groups. $\mathrm{p}<0.05$ was considered to be significant.

\section{Results}

\section{Iron chelator DFO decreases tumorigenicity in vivo}

Based on the epidemiological evidences that cancer patient links with an increased body iron storage 28 , so we used Deferoxamine (DFO), a chelator used in clinic which could chelate iron efficiently, to explore whether iron was involved in the tumorigenesis of osteosarcoma in vivo (Fig. 1e). DFO group showed a smaller tumor volume and lower weight compared with control group (Fig. 1a-c). Moreover, DFO treatment led to a lower serum iron level when compared to control group (Fig. 1d). These results indicated that DFO could inhibit the development of osteosarcoma, which implied that iron maybe was involved in the tumorigenesis of osteosarcoma.

\section{Iron promotes osteosarcoma cell lines proliferation and affects cell cycle progression}

Two osteosarcoma cell lines SAOS-2 and U2OS were treated with exogenous iron (ferric ammonium citrate, FAC), DFO or PBS at indicated times. We found FAC could increase the viability of both cell lines (Fig. 2a). Flow cytometry analysis revealed that FAC treatment increases the percentage of $S$ phase and decreases the percentage of G1 phase. Whereas DFO treatment presented as na inverse results, which indicated that iron affects cell cycle progression (Fig. 2b). The colony formation assay showed an increase of colony number in FAC group, whereas DFO group showed a decrease of colony number compared to control group (Fig. 2c). The results of EdU cell proliferation assay showed an increase of cell percentage in cells treated with FAC (Fig. 2d, e). Together, these results demonstrated that the cell proliferative ability and cell cycle progression could be promoted by FAC while DFO inhibit these processes, indicating that iron could be a promoter in osteosarcoma cell lines proliferation. Proliferating cell nuclear antigen (PCNA) was reported to be an indicator in cell proliferation. We found that iron increased PCNA protein level while DFO showed an inverse result (Fig. 2f).

\section{Iron promotes osteosarcoma carcinogenesis and increases} migration and invasion ability of both cells

The soft-agar colony formation assay was used to detect the carcinogenesis of osteosarcoma. Colony number of FAC-treated showed a significant increase compared to other groups (Fig. 3a). In the trans-well assay, FAC group showed more SAOS-2 and U2OS cells than any other groups (Fig. 3b). The wound healing assay, FAC group showed a smaller wound area (Fig. 3c). Collectively, these results indicated iron promotes carcinogenesis, invasion and migration ability of both osteosarcoma cells carcinogenesis, invasion and migration.

\section{Mitochondrion-mediated iron accumulation promotes Warburg effect}

Warburg effect, as one of the hallmarks of cancer, presented as a metabolic disorder. Even in oxygen condition cancer cells acquire energy by aerobic glycolysis. Seahorse metabolic analyzer is used to detect the extracellular acidification (ECAR) and oxygen consumption rates (OCR). FAC-treated group showed a higher ECAR compared to that of control group, whereas DFO-treated 


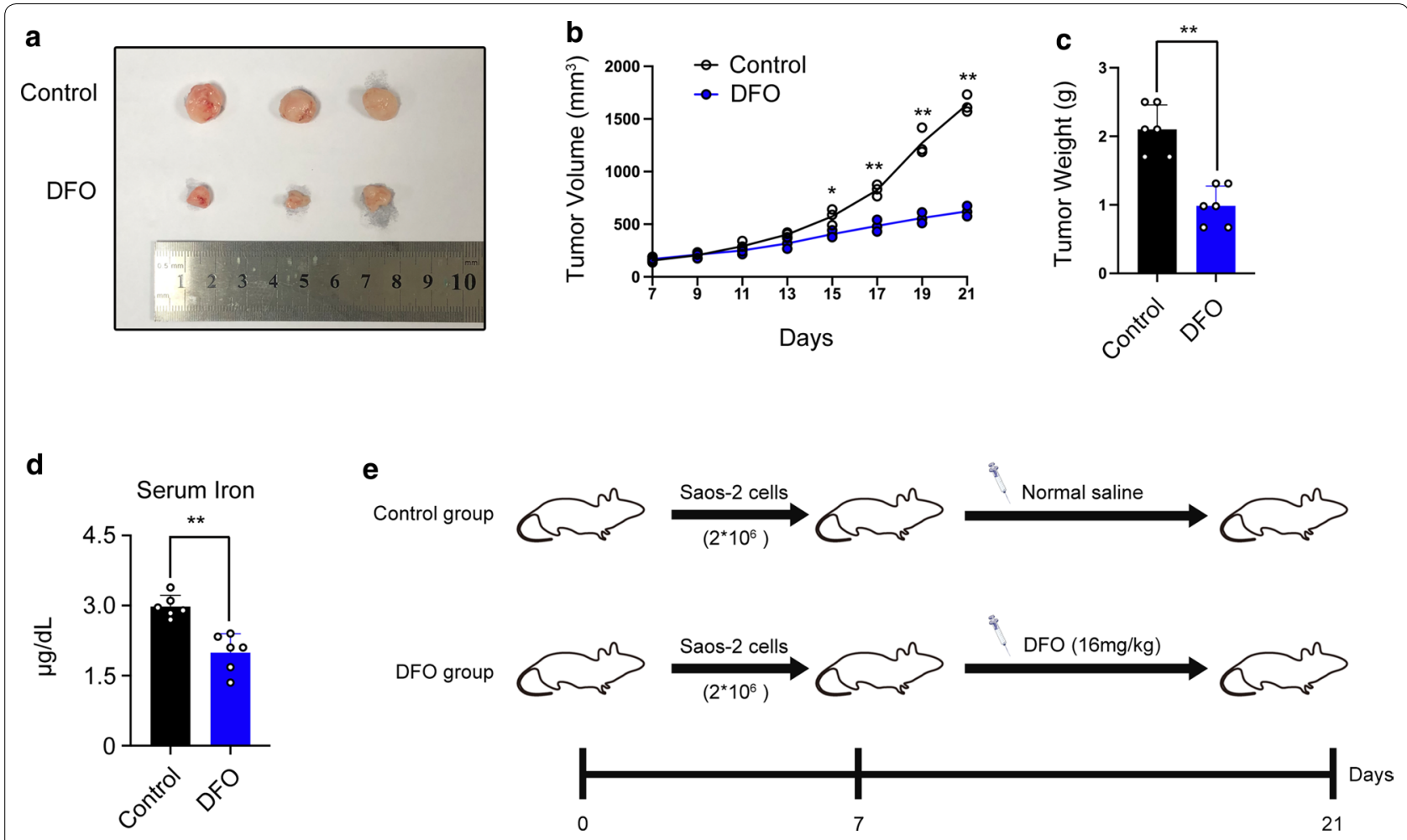

Fig. 1 Deferoxamine (DFO) inhibited osteosarcoma development. a Representative images of tumors injected with $2 * 10^{6}$ SAOS-2 cells subcutaneously $(n=6)$. b Tumor volumes were measured by the indicated time $(n=6)$. c Tumor weight $(n=6)$. d Serum iron analyses $\left(^{*} p<0.05\right.$, ${ }^{* *} \mathrm{p}<0.01$, mean $\left.\pm S D, n=6\right)$. e Proposed scheme of animal experiment

group showed a reduced ECAR. OCR results in FAC group were significantly lower than other groups in both cell lines (Fig. 4a-d). We further wanted to investigate the places where iron accumulated in cells. Iron assay was used to detect the iron content in cytoplasm and mitochondrion according to the instruction of Iron Assay Kit. Mitochondrion to cytoplasm ratio indicated that iron was accumulated more in mitochondrion than that of cytoplasm (Fig. 4e). Next, mitochondrial iron importer proteins mitoferrin 1 (SLC25A37) and mitoferrin 2 (SLC25A28) were detected by western blot. We found FAC could increase the expression of mitoferrin 1 (SLC25A37) and mitoferrin 2 (SLC25A28) (Fig. 4f). Taken together, these results showed that iron promoted Warburg effect of osteosarcoma cells through the mitochondrion-mediated iron accumulation.

\section{Mitochondrion-mediated iron accumulation increases ROS production by mitoferrin}

Studies have shown that iron could increase ROS production and promote carcinogenesis $[20,21]$. We then explored ROS production in each group by DCFH$\mathrm{DA}$, and then found that FAC-treated group with a significant increase of ROS. A significant ROS inhibition was observed when ROS scavenger NAC (5 mM) was added into FAC culture medium for $2 \mathrm{~h}$. Interestingly, DFO exhibited as a ROS scavenger (Fig. 4g). Next, we wanted to explore the underlying mechanisms of ironmediated ROS production. Depletion of SLC25A37 and SLC25A28 by RNAi could significantly reduce ROS production when cells treated by FAC (Fig. 4h). The expression of shRNA was confirmed by western blot and qPCR Additional file 2: Fig. S1). Collectively, our results demonstrated that iron could increase ROS production by mitoferrin 1 (SLC25A37) and mitoferrin 2 (SLC25A28).

\section{SLC25A37 had a positive correlation with key genes of Warburg effect}

Based on the results described above, GEPIA 2 online tools which based on TCGA database, was used to investigate the correlation of SLC25A37 and Warburg key genes including HK2, GLUT1, GAPDH, PGK1, ENO1, PKM and LDHA (http://gepia2.cancer-pku.cn/\#corre lation). GEPIA (Gene Expression Profiling Interactive Analysis) web server, which was based on tumor and normal samples from the TCGA and the GTEx databases, has been a valuable and highly cited resource for gene expression analysis since introduced in 2017. In addition, 


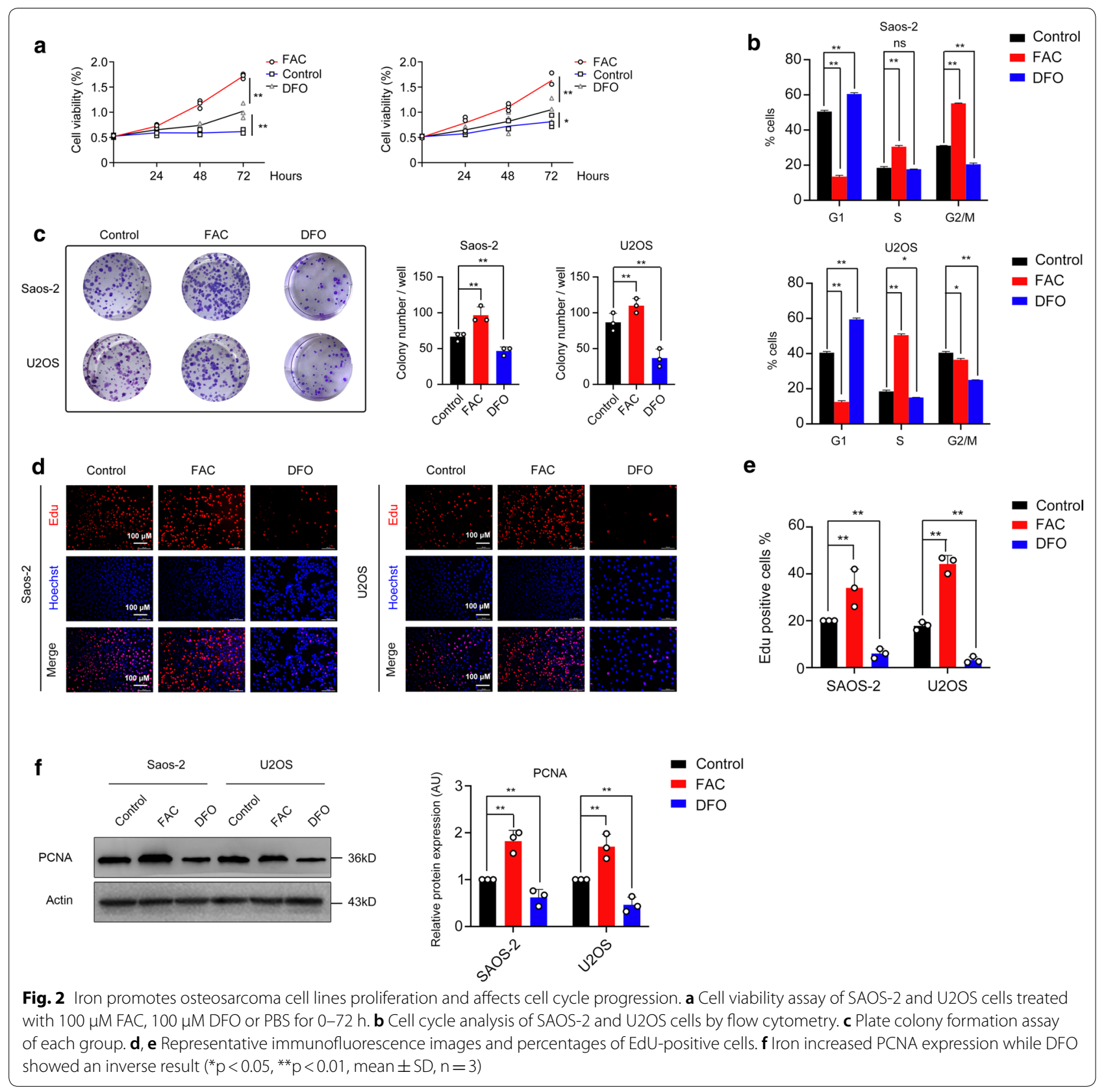

(See figure on next page.)

Fig. 3 Iron promoted carcinogenesis, migration and invasion of osteosarcoma cell-lines SAOS-2 and U2OS. a Soft-agar colony formation assay showing ferric ammonium citrate (FAC) promoted the growth of osteosarcoma cells. b Ferric ammonium citrate (FAC) increased the migration and invasion of SAOS-2 and U2OS cells. c Ferric ammonium citrate (FAC) showed a smaller wound area after $24 \mathrm{~h}$ incubation $\left({ }^{*} \mathrm{p}<0.05,{ }^{* *} \mathrm{p}<0.01\right.$, mean $\pm S D, n=3$ )

GEPIA2 has adopted new analysis techniques of gene signature quantification inspired by single-cell sequencing studies, and provides customized analysis where users can upload their own RNA-seq data and compare them with TCGA and GTEx samples. $\mathrm{p}<0.05$ was considered as statistically significant by using GEPIA2 web tools. HK2, GLUT1, PGK1, ENO1 and PKM were found 


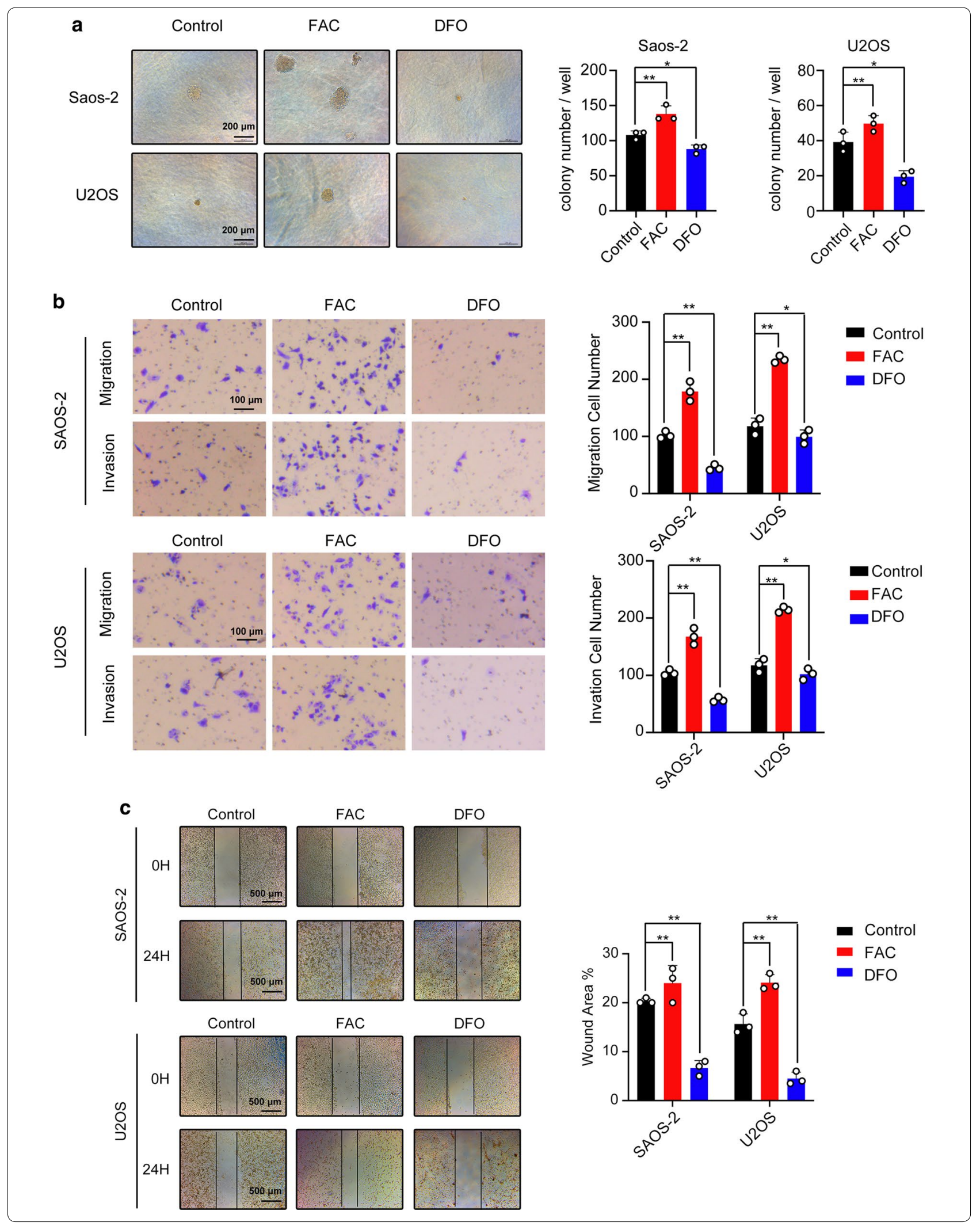



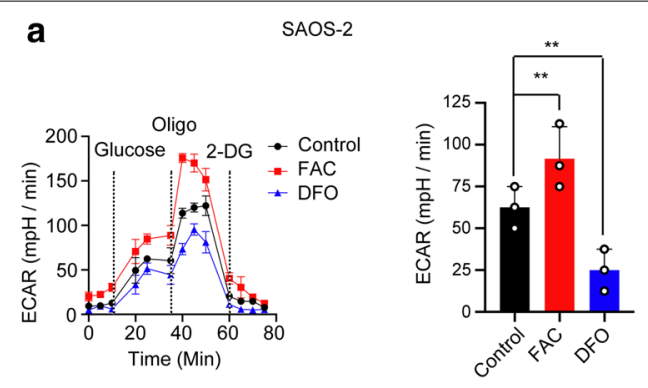

c

U2OS
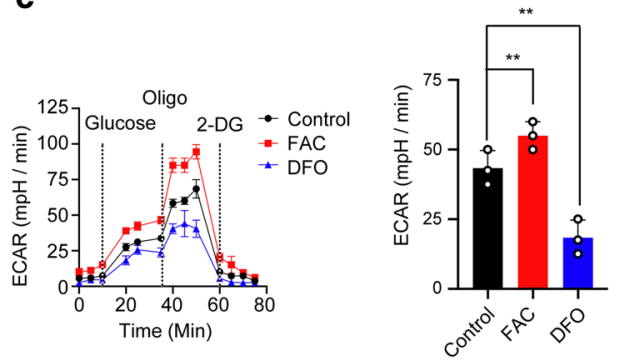
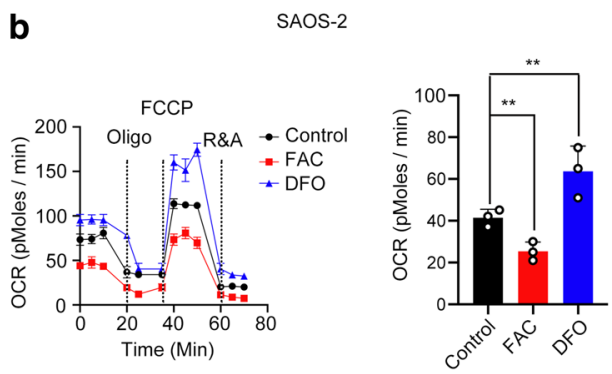

d

U2OS

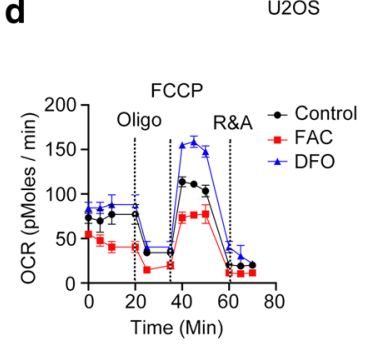

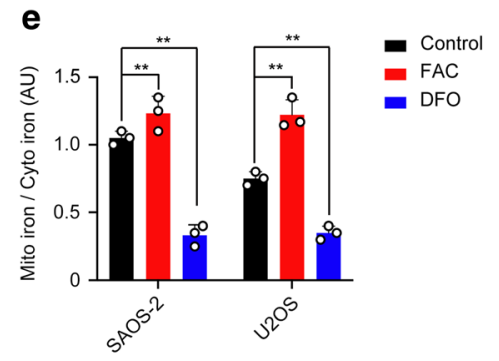
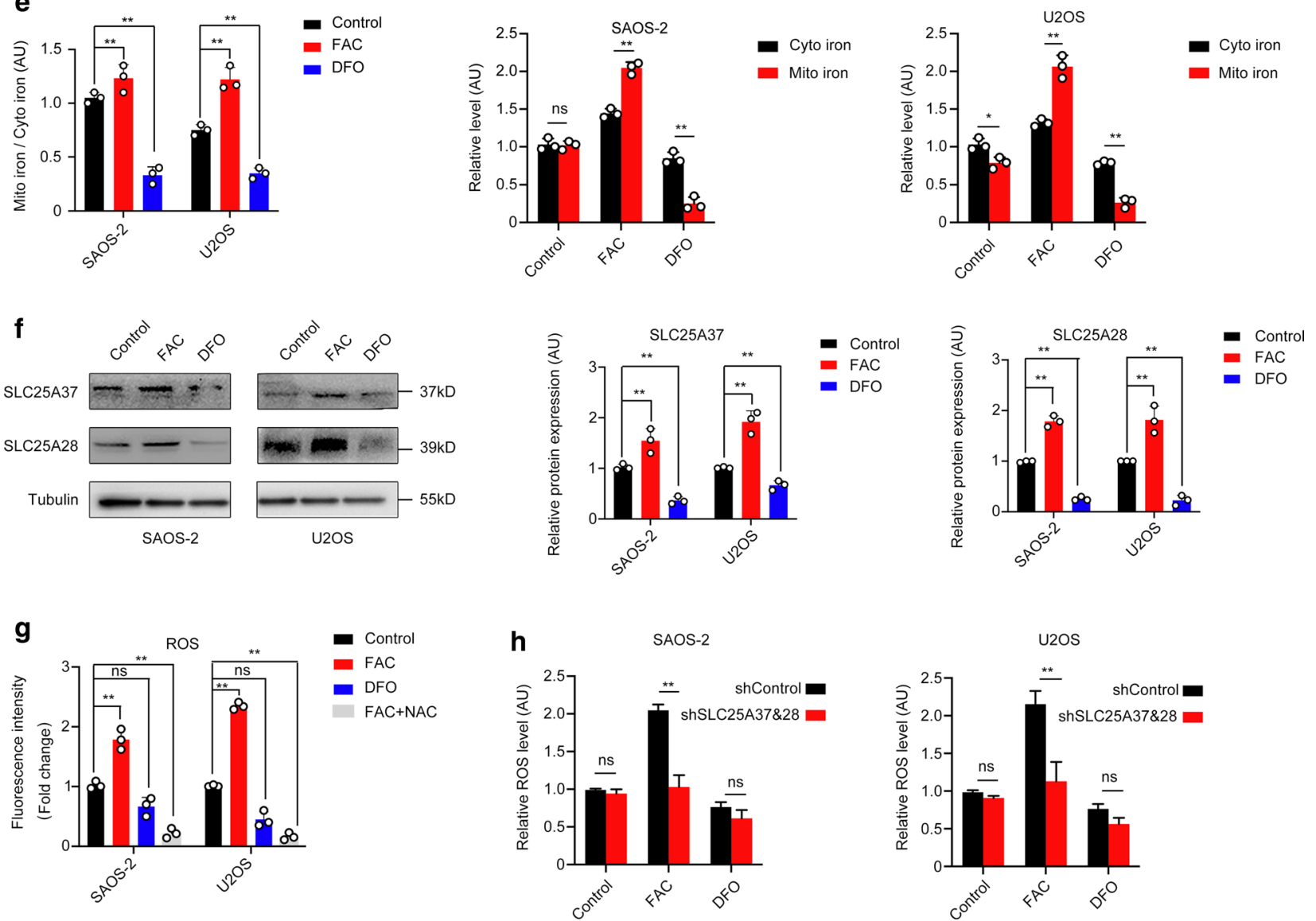

Fig. 4 Mitochondrion-mediated iron accumulation promotes Warburg effect through reactive oxygen species. a-d Seahorse metabolic analysis of extracellular acidification (ECAR) and oxygen consumption rates (OCR) in both cell lines. e Mitochondrion iron accumulation was higher than that of cytoplasm. f Ferric ammonium citrate (FAC) increased mitoferrin 1 (SLC25A37) and mitoferrin 2 (SLC25A28) expression. g Ferric ammonium citrate (FAC) increased reactive oxygen species (ROS) production. $\mathbf{h}$ Depletion of mitoferrin 1 (SLC25A37) and mitoferrin 2 (SLC25A28) by shRNA rescued the ROS production by FAC $\left({ }^{*} p<0.05,{ }^{* *} p<0.01\right.$, ns: no significant, mean $\pm S D, n=3$ ) 
positive correlation with SLC25A37 in sarcoma patients (Fig. 5a).

\section{Iron increased Warburg key protein HK2 and GLUT1 expression, which were associated with poor prognosis in osteosarcoma patients}

According to the results we found by GEPIA 2 online tools and several studies reported, the expression of Warburg key proteins HK2 and Glut1 were significantly increased compared to control group, while DFO rescued the expression of HK2 and Glut1 (Fig. 5b). Further, we evaluated the correlations between those protein expressions and the survival of sarcoma patients. A number of 131 cases of sarcoma was analyzed and we found that HK2 and Glut1 high expression associated with poor prognosis in osteosarcoma patients, $\mathrm{p}=0.023$ and $p=0.013$, respectively (Fig. $5 \mathrm{c}$ ). Given the fact that we are unable to collect osteosarcoma specimen due to the lack of such patients in our hospital, so we didn't perform those genes expression in clinic as other study reported [29].

\section{Iron may affect AMPK and subsequently promote mTORC1 activity}

AMP-activated protein kinase (AMPK) worked as a regulator of cell metabolism and a sensor of energy, energy stress-induced activation of AMPK inhibits the activation of mTORC1, and mTORC1 is activated in the inverse pattern [30]. We next investigated whether this pathway is involved in iron-induced metabolism abnormal. The inhibition of $\mathrm{p}$-AMPK and subsequently the activation of mTORC1 was observed (Fig. 6a, b). Moreover, iron-induced activation of p-AMPK was observed in a dose-dependent manner (FAC $10 \mu \mathrm{M}$ and $100 \mu \mathrm{M}$ ). Taken together, we found iron may affect AMPK and subsequently promote mTORC1 activity.

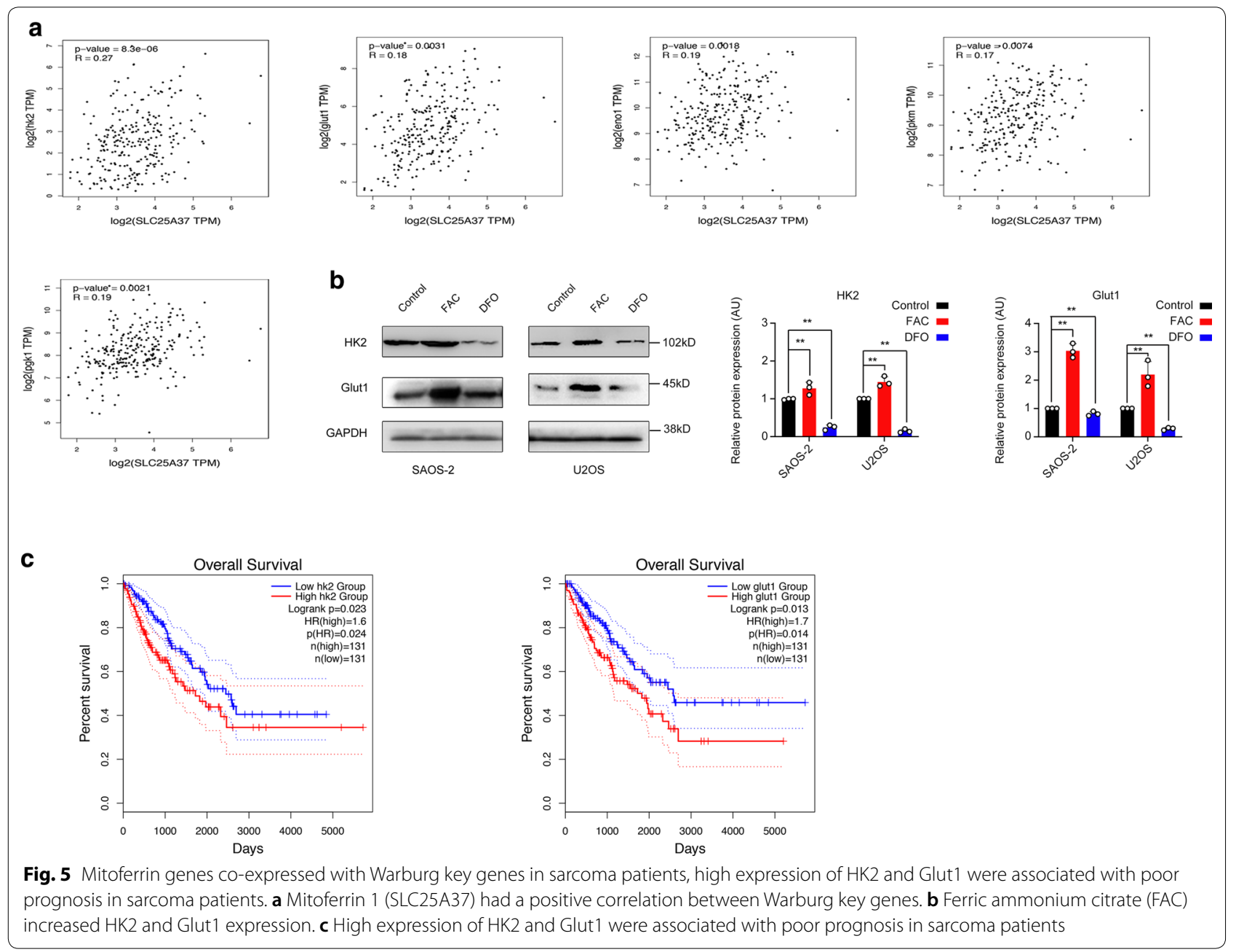




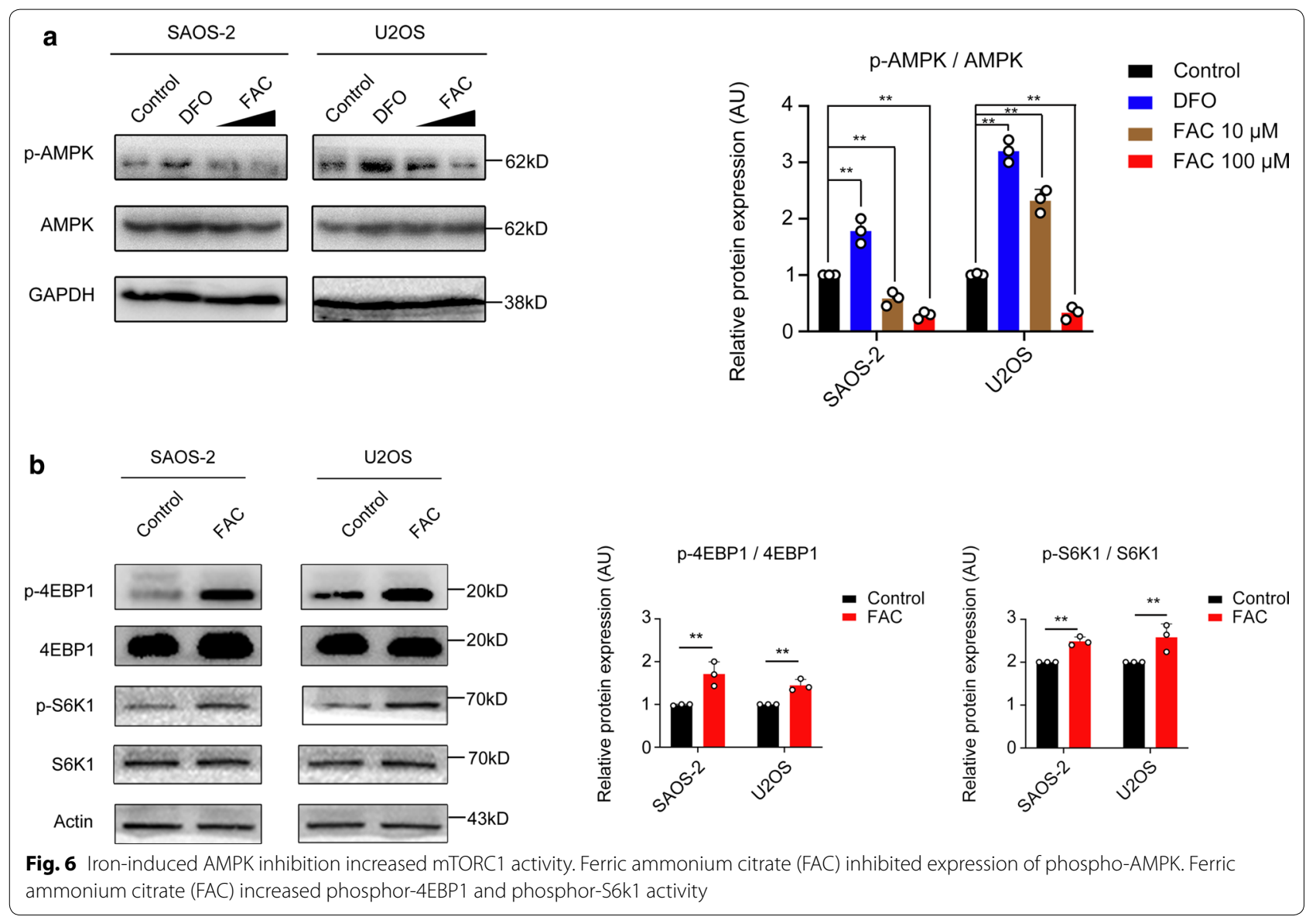

\section{Discussion}

Numerous evidences have demonstrated that iron is associated with the initiation and development of several malignant tumors $[17,19,21]$. Iron overload promotes carcinogenesis and tumorigenesis in colorectal and lung cancer [17-19]. Moreover, several studies have also demonstrated the therapeutic effects of iron chelator in lung cancer, pancreatic cancer, multiple myeloma and colorectal cancer $[15,17-19,21,28]$. In the present study, we generated a tumor-load model by injecting SAOS-2 cell line in nude mice. After 14 days of DFO treatment, tumor volume and weight were significantly reduced. These results implied that iron maybe a promoter in osteosarcoma. Based on the epidemiological evidences that cancer patient showed with an increased body iron storage [28], so we do not set a iron-treated group in animal experiment though. To our surprise, iron chelator DFO could significantly reduce the tumor volume and weight, which implied that iron maybe a promoter in the development of osteosarcoma.

The mechanisms underlying iron and the carcinogenesis in osteosarcoma are not yet well understood. Studies have reported that intracellular iron excess resulted in the generation of ROS, degradation of P53, and the activation of gp130/STAT3 signaling pathway [17]. Iron-mediated ROS promotes Kras-driven pancreatic tumorigenesis [21]. Ferroportin-mediated ROS up-regulation promotes the proliferation of multiple myeloma [31]. Studies have also revealed that ROS can affect cell metabolism and mitochondrial function, thus influencing Warburg effect [32]. In our work, we found that mitochondrion-mediated iron accumulation played an important role in the development of osteosarcoma through ROS. Further, mitochondrion-mediated iron accumulation contributed to the proliferation, migration and invasion of osteosarcoma, as well as the Warburg effect by ROS.

Warburg effect, as one of the hallmarks of cancer, presented as a metabolic disorder. Warburg effect is associated closely with proliferation, metastasis and drug resistance of cancer cell. In our study, seahorse metabolic analysis showed a higher aerobic glycolysis and a decrease of oxygen consumption rate, a classical Warburg phenotype, which implied that iron could be a factor in this process. Several studies have demonstrated that reactive oxygen species (ROS) regulate Warburg effect directly or indirectly [21, 24-26]. In normal cells, 
ROS production is mainly from mitochondrion intracellular [33]. Interestingly, our work revealed that mitochondrion-mediated iron accumulation increased ROS production in both osteosarcoma cell lines, whereas knock-down of mitoferrin 1 (SLC25A37) and mitoferrin 2 (SLC25A28) decreased this phenomenon. Accordingly, our work proved that mitochondrion-mediated iron accumulation played a critical role in Warburg effect in osteosarcoma. We further explored the correlation between mitoferrin genes and Warburg key genes. Surprisingly, mitoferrin 1 (SLC25A37) had a positive correlation with HK2, GLUT1, PGK1, ENO1 and PKM, whereas mitoferrin 2 (SLC25A28) had a negative correlation with those genes. Moreover, we found that the expression of HK2 and GLUT1 was increased in iron-treated group. These results suggested that iron maybe a risk factor, which increases the expression of Warburg key genes of sarcoma patients. Given the fact that we are unable to collect osteosarcoma specimen due to the lack of such patients in our hospital, so we didn't perform those genes expression by WB or IHC staining.

AMP-activated protein kinase (AMPK) worked as a regulator of cell metabolism and a sensor of energy, it could activate the mTOR complex 1 (mTORC1) signaling pathway when AMPK is inhibited. According to our previous results, iron may promote the proliferation of SAOS-2 and U2OS cell lines in vitro. Moreover, iron may promote the Warburg effect according to Seahorse metabolic analysis, all of which made us believe that the energy consumption of two osteosarcoma cell lines was improved significantly when iron was added into the culture medium. In addition, an improvement of aerobic glycolysis in osteosarcoma cell lines, which confirmed by Seahorse, resulting in an abundance of ATP and the essential materials of cells needed in cytoplasm, making osteosarcoma cells in a nutrition-rich condition thus rendering the inactive of AMPK as other study reported [30]. Further, since energy stress-induced activation of AMPK is also known to inhibit the activation of mTORC1, and mTORC1 is activated in the inverse pattern [34]. PI3kAKT-mTOTC axis is an effective alternative proved by several clinical trials in the treatment of cancer [35]. Recently, study has found the inhibition of HK2 activated AMPK and thus suppressed its down-stream mTORC1 in lung cancer [34]. We further investigated the AMPKmTORC1 signaling axis at the end of our work. The inhibition of p-AMPK and activation of its down-stream mTORC1, as indicated by the level of S6K1 (Thr389) and 4EBP1 (Thr70) phosphorylation, was observed when osteosarcoma cells treated with iron. Taken together, we demonstrated that iron could affect osteosarcoma cells metabolism by ROS and AMPK signaling pathway. These findings, in turn, suggested that iron chelator could be an effective therapeutic alternative in osteosarcoma.

\section{Conclusions}

Our results highlighted the iron-induced carcinogenesis of osteosarcoma, and proved that mitochondrion-mediated iron accumulation promotes carcinogenesis and Warburg effect of osteosarcoma through reactive oxygen species. These findings may also provide some clues to elucidating the underlying mechanism of iron-dependent disease, especially in osteosarcoma. These findings in turn suggested that iron chelator maybe a therapeutic alternative in osteosarcoma.

\section{Supplementary information}

Supplementary information accompanies this paper at https://doi. org/10.1186/s12935-020-01494-3.

Additional file 1: Table S1. Primers used in the real-time PCR. Table S2. Primers used in shRNA

Additional file 2: Figure S1. Depletion of mitoferrin 1 (SLC25A37) and mitoferrin 2 (SLC25A28) were tested by Western blot and qPCR. a. Western blot showed the expression of mitoferrin 1 (SLC25A37) and mitoferrin 2 (SLC25A28) on protein level. b. qPCR results of depletion of mitoferrin 1 (SLC25A37) and mitoferrin 2 (SLC25A28) by shRNA. $\left({ }^{*} \mathrm{p}<0.05\right.$, ${ }^{* *} \mathrm{p}<0.01$, ns: no significant, mean $\pm S D, n=3$ )

\section{Abbreviations}

DMT1: Divalent metal transporter 1; FPN1: Ferroportin 1; ROS: Reactive oxygen species; FAC: Ferric ammonium citrate; DFO: Deferoxamine; DCFH-DA: Dichlorodihydrofluorescein diacetate; OCR: Oxygen consumption rate; ECAR : Extracellular acid rate; AMPK: AMP-activated protein kinase; mTORC1: mTOR complex 1.

\section{Acknowledgements}

The authors are indebted to Dr. Dejian Li (Fudan university Pudong Medical Center), Dr. Haibo Du (Donghua University) and Dr. Zhanrong Kang (Shanghai Pudong Hospital) for the generous gift of SAOS-2 cell line.

\section{Authors' contributions}

$\mathrm{SN}$, YK and YY contributed equally to this work. All authors read and approved the final manuscript.

\section{Funding}

This work was supported by the Natural Science Foundation of China (Project no. 81971753), Program for Medical Key Department of Shanghai (Grant No. ZK2019C01), The Outstanding Clinical Discipline Project of Shanghai Pudong (Grant No. PWYgy2018-09), The fund of Key Disciplines Group Construction Project of Pudong Health Bureau of Shanghai (Grant No. PWZxq2017-11), Program for Outstanding Leader of Shanghai (Grant No. 046).

\section{Availability of data and materials}

The datasets used and/or analyzed during the current study are available from the corresponding author upon reasonable request.

\section{Ethics approval and consent to participate}

The animal experiments in this study was approved by the Animal Ethics Committee of Zhejiang University School of Medicine, in accordance with the National Institutes of Health (NIH) Guide for the animal treatments of Laboratory Animals.

\section{Consent for publication}

Not applicable. 


\section{Competing interests}

The authors declare that they have no competing interests.

\section{Author details}

1 Department of Orthopedics, Shanghai Pudong Hospital, Fudan University Pudong Medical Center, 2800 Gongwei Road, Pudong, Shanghai 201399, China. ${ }^{2}$ Department of Respiratory Medicine, School of Medicine, Ren Ji Hospital, Shanghai Jiao Tong University, Shanghai 200127, China. ${ }^{3}$ State Key Laboratory for Diagnosis and Treatment of Infectious Diseases, The First Affiliated Hospital, School of Medicine, Zhejiang University, Hangzhou 310003, China.

Received: 7 May 2020 Accepted: 11 August 2020

Published online: 18 August 2020

\section{References}

1. Arredondo M, Núñez MT. Iron and copper metabolism. Mol Aspects Med. 2005;26(4-5):313-27.

2. Heath JL, Weiss JM, Lavau CP, Wechsler DS. Iron deprivation in cancerpotential therapeutic implications. Nutrients. 2013;5(8):2836-59.

3. Nairz M, Schroll A, Demetz E, Tancevski I, Theurl I, Weiss G. 'Ride on the ferrous wheel'-the cycle of iron in macrophages in health and disease. Immunobiology. 2015;220(2):280-94.

4. Hentze MW, Muckenthaler MU, Galy B, Camaschella C. Two to tango: regulation of Mammalian iron metabolism. Cell. 2010;142(1):24-38.

5. Bach FH. Heme oxygenase-1: a therapeutic amplification funnel. FASEB J. 2005;19(10):1216-9.

6. Gozzelino R, Andrade BB, Larsen R, et al. Metabolic adaptation to tissue iron overload confers tolerance to malaria. Cell Host Microbe. 2012;12(5):693-704.

7. Lill R. Function and biogenesis of iron-sulphur proteins. Nature. 2009:460(7257):831-8.

8. Paradkar PN, Zumbrennen KB, Paw BH, Ward DM, Kaplan J. Regulation of mitochondrial iron import through differential turnover of mitoferrin 1 and mitoferrin 2. Mol Cell Biol. 2009;29(4):1007-16.

9. Ichikawa Y, Ghanefar M, Bayeva M, et al. Cardiotoxicity of doxorubicin is mediated through mitochondrial iron accumulation. J Clin Invest. 2014;124(2):617-30.

10. Haddad S, Wang Y, Galy B, et al. Iron-regulatory proteins secure iron availability in cardiomyocytes to prevent heart failure. Eur Heart J. 2017;38(5):362-72.

11. Cloonan SM, Glass K, Laucho-Contreras ME, et al. Mitochondrial iron chelation ameliorates cigarette smoke-induced bronchitis and emphysema in mice. Nat Med. 2016;22(2):163-74.

12. Zacharski LR, Chow BK, Howes PS, et al. Decreased cancer risk after iron reduction in patients with peripheral arterial disease: results from a randomized trial. J Natl Cancer Inst. 2008;100(14):996.

13. Osborne NJ, Gurrin LC, Allen KJ, et al. HFE C282Y homozygotes are at increased risk of breast and colorectal cancer. Hepatology. 2010;51(4):1311-8.

14. Torti SV, Manz DH, Paul BT, Blanchette-Farra N, Torti FM. Iron and Cancer. Annu Rev Nutr. 2018:38:97-125.

15. Nelson RL. Iron and colorectal cancer risk: human studies. Nutr Rev. 2001;59(5):140-8

16. Xue X, Taylor M, Anderson E, et al. Hypoxia-inducible factor-2a activation promotes colorectal cancer progression by dysregulating iron homeostasis. Cancer Res. 2012;72(9):2285-93.
17. Kuang $Y$, Wang Q. Iron and lung cancer. Cancer Lett. 2019;464:56-61.

18. Xue X, Ramakrishnan SK, Weisz K, et al. Iron uptake via DMT1 integrates cell cycle with JAK-STAT3 signaling to promote colorectal tumorigenesis. Cell Metab. 2016;24(3):447-61.

19. Kuang Y, Guo W, Ling J, et al. Iron-dependent CDK1 activity promotes lung carcinogenesis via activation of the GP130/STAT3 signaling pathway. Cell Death Dis. 2019:10(4):297.

20. Seril DN, Liao J, Yang G-Y, Yang CS. Oxidative stress and ulcerative colitis-associated carcinogenesis: studies in humans and animal models. Carcinogenesis. 2003;24(3):353-62.

21. Li C, Zhang $Y$, Cheng $X$, et al. PINK1 and PARK2 suppress pancreatic tumorigenesis through control of mitochondrial iron-mediated immunometabolism. Dev Cell. 2018;46(4):441.

22. Li P, Zheng X, Shou K, et al. The iron chelator Dp44mT suppresses osteosarcoma's proliferation, invasion and migration: in vitro and in vivo. Am J Transl Res. 2016;8(12):5370-85.

23. Shang C, Zhou H, Liu W, Shen T, Luo Y, Huang S. Iron chelation inhibits mTORC1 signaling involving activation of AMPK and REDD1/Bnip3 pathways. Oncogene. 2020;39(29):5201-13.

24. Agnihotri S, Golbourn B, Huang X, et al. PINK1 is a negative regulator of growth and the Warburg effect in glioblastoma. Cancer Res. 2016:76(16):4708-19.

25. Teng R, Liu Z, Tang H, et al. HSP60 silencing promotes Warburg-like phenotypes and switches the mitochondrial function from ATP production to biosynthesis in ccRCC cells. Redox Biol. 2019;24:101218.

26. Hart PC, Mao M, de Abreu ALP, et al. MnSOD upregulation sustains the Warburg effect via mitochondrial ROS and AMPK-dependent signalling in cancer. Nat Commun. 2015;6:6053.

27. Alessio N, Aprile D, Squillaro T, et al. The senescence-associated secretory phenotype (SASP) from mesenchymal stromal cells impairs growth of immortalized prostate cells but has no effect on metastatic prostatic cancer cells. Aging. 2019;11(15):5817-28.

28. Torti SV Torti FM. Iron and cancer: more ore to be mined. Nat Rev Cancer. 2013;13(5):342-55.

29. Finicelli M, Benedetti G, Squillaro T, et al. Expression of stemness genes in primary breast cancer tissues: the role of SOX2 as a prognostic marker for detection of early recurrence. Oncotarget. 2014;5(20):9678-88.

30. Shaw RJ. LKB1 and AMP-activated protein kinase control of mTOR signalling and growth. Acta Physiol (Oxf). 2009;196(1):65-80.

31. Kong $Y$, Hu L, Lu K, et al. Ferroportin downregulation promotes cell proliferation by modulating the Nrf2-miR-17-5p axis in multiple myeloma. Cell Death Dis. 2019:10(9):624.

32. Rodic S, Vincent MD. Reactive oxygen species (ROS) are a key determinant of cancer's metabolic phenotype. Int J Cancer. 2018;142(3):440-8.

33. Turrens JF. Superoxide production by the mitochondrial respiratory chain. Biosci Rep. 1997:17(1):3-8

34. Guo W, Kuang Y, Wu J, et al. Hexokinase 2 depletion confers sensitization to metformin and inhibits glycolysis in lung squamous cell carcinoma. Front Oncol. 2020;10:52

35. Momcilovic M, Bailey ST, Lee JT, et al. The GSK3 signaling axis regulates adaptive glutamine metabolism in lung squamous cell carcinoma. Cancer Cell. 2018;33(5):905-21.

\section{Publisher's Note}

Springer Nature remains neutral with regard to jurisdictional claims in published maps and institutional affiliations. 